\title{
Polarimetric purity and the concept of degree of polarization
}

\author{
José J. Gil, ${ }^{1,{ }^{*}}$ Andreas Norrman, ${ }^{2}$ Ari T. Friberg, ${ }^{3}$ and Tero Setälä ${ }^{3}$ \\ ${ }^{1}$ Facultad de Educación, Universidad de Zaragoza, Pedro Cerbuna 12, 50009 Zaragoza, Spain \\ ${ }^{2}$ Max Planck Institute for the Science of Light, Staudtstraße 2, D-91058 Erlangen, Germany \\ ${ }^{3}$ Institute of Photonics, University of Eastern Finland, P.O. Box 111, FI-80101 Joensuu, Finland
}

(Received 3 July 2017; published 26 February 2018)

\begin{abstract}
The concept of degree of polarization for electromagnetic waves, in its general three-dimensional version, is revisited in the light of the implications of the recent findings on the structure of polarimetric purity and of the existence of nonregular states of polarization [J. J. Gil et al., Phys Rev. A 95, 053856 (2017)]. From the analysis of the characteristic decomposition of a polarization matrix $\mathbf{R}$ into an incoherent convex combination of (1) a pure state $\mathbf{R}_{p}$, (2) a middle state $\mathbf{R}_{m}$ given by an equiprobable mixture of two eigenstates of $\mathbf{R}$, and (3) a fully unpolarized state $\mathbf{R}_{u-3 \mathrm{D}}$, it is found that, in general, $\mathbf{R}_{m}$ exhibits nonzero circular and linear degrees of polarization. Therefore, the degrees of linear and circular polarization of $\mathbf{R}$ cannot always be assigned to the single totally polarized component $\mathbf{R}_{p}$. It is shown that the parameter $P_{3 \mathrm{D}}$ proposed formerly by Samson [J. C. Samson, Geophys. J. R. Astron. Soc. 34, 403 (1973)] takes into account, in a proper and objective form, all the contributions to polarimetric purity, namely, the contributions to the linear and circular degrees of polarization of $\mathbf{R}$ as well as to the stability of the plane containing its polarization ellipse. Consequently, $P_{3 \mathrm{D}}$ constitutes a natural representative of the degree of polarimetric purity. Some implications for the common convention for the concept of two-dimensional degree of polarization are also analyzed and discussed.
\end{abstract}

DOI: 10.1103/PhysRevA.97.023838

In the space-time representation, polarization of electromagnetic waves refers to the temporal evolution of the electric field vector at a given fixed point in the space. A frequent assumption in polarization optics is that the polarization ellipse remains in a fixed plane during the measurement time [in which case, the state of polarization is said to be a two-dimensional (2D) state]. Obviously, this situation is not general because the electric field of the electromagnetic wave evolves in the real three-dimensional (3D) space and therefore a general mathematical description of the states of polarization requires a 3D treatment $[1,2]$. The necessity of developing appropriate mathematical formalisms to describe 3D polarization phenomena has been intensified in the recent years due to the increasing attention paid to near-field phenomena and nanotechnologies. One of the more significant notions in polarization theory is the degree of polarization, which requires an appropriate definition beyond the common convention used for $2 \mathrm{D}$ states. In the case of $2 \mathrm{D}$ states of polarization, it is always possible to represent them as an incoherent convex composition of a pure state (i.e., fully polarized) and an unpolarized state [3,4]. In general, however, this kind of decomposition cannot be extended to 3D states [1,5]. Therefore, the notion of a 3D degree of polarization must be addressed from different points of view.

A possible approach is based on identifying the totally polarized component of the given $3 \mathrm{D}$ polarization state and defining the degree of polarization $\left(P_{1}\right)$ as the ratio between its intensity and that of the whole state [6,7]. Clearly, this idea matches perfectly the common definition of the degree of polarization for $2 \mathrm{D}$ states. An alternative approach is obtained

\footnotetext{
*ppgil@unizar.es
}

by identifying a quantity $\left(P_{3 \mathrm{D}}\right)$ which satisfies the requirement that it takes on the value 0 for states with fully random polarization (unpolarized 3D states) and monotonically varies up to the value 1 for pure states (fully polarized states) [8,9]. Between the two extremes $P_{3 \mathrm{D}}$ characterizes, in analogy to the $2 \mathrm{D}$ degree of polarization [3], the strength of correlations among orthogonal field components [8].

The main aim of this paper is to analyze and compare the above-mentioned approaches in the light of certain recent results dealing with the existence of regular and nonregular polarization states [10]. In particular, we consider the contributions of their linear and circular degrees of polarization to polarimetric purity. We demonstrate that $P_{1}$ fails to account for all such contributions, whereas $P_{3 \mathrm{D}}$ contains, in a meaningful way, all the purity contributions including the stability of the plane of the polarization ellipse. Hence, we conclude that the quantity $P_{3 \mathrm{D}}$ constitutes a proper and natural representative for the notion of degree of polarimetric purity.

We begin by summarizing the necessary theoretical background. A totally polarized, or pure, state is characterized by the fact that its polarization ellipse remains in a fixed plane and maintains its shape (but not necessarily its size [11]) during the measurement time considered. For the sake of clarity of further analyses, it is worth recalling that any pure state is represented by a polarization matrix $\mathbf{R}_{p}$ of rank $\mathbf{R}_{p}=1$, which can always be written as $\mathbf{R}_{p} \equiv\left(\operatorname{tr} \mathbf{R}_{p}\right)\left(\mathbf{w} \otimes \mathbf{w}^{\dagger}\right)$ where tr denotes trace, $\otimes$ stands for the Kronecker product, the superscript $\dagger$ indicates the conjugate transpose, and $\mathbf{w}$ is the corresponding three-dimensional complex unit vector. Two pure states $\mathbf{R}_{p 1} \equiv\left(\operatorname{tr} \mathbf{R}_{p 1}\right)\left(\mathbf{w}_{1} \otimes \mathbf{w}_{1}^{\dagger}\right)$ and $\mathbf{R}_{p 2} \equiv\left(\operatorname{tr} \mathbf{R}_{p 2}\right)\left(\mathbf{w}_{2} \otimes \mathbf{w}_{2}^{\dagger}\right)$ are mutually orthogonal when their respective unit vectors $\mathbf{w}_{1}$ and $\mathbf{w}_{2}$ satisfy $\mathbf{w}_{1}^{\dagger} \mathbf{w}_{2}=\delta_{12}$, where $\delta_{12}$ is the Kronecker 
delta. Polarimetric purity of an arbitrary 3D state refers to the degree of polarimetric determinism; i.e., it is a measure of the closeness of the polarization state to a fully polarized one [9,11]. Alternatively, polarimetric purity may be viewed as an overall measure of how mutually correlated the components of the field are.

Obviously, regardless of the fact that usually the plane containing the polarization ellipse, determined by the evolution of the end point of the electric field vector of an electromagnetic wave, is considered stable (2D states of polarization), any polarization state is realized in the three-dimensional (3D) real space. Under the second-order approach (i.e., leaving aside moments of the analytic signals of the field variables of order higher than 2), the polarization (or coherency) matrix $\mathbf{R}$ is a positive semidefinite Hermitian matrix that fully characterizes the polarization properties at a single point. $\mathbf{R}$ can always be expressed as

$$
\begin{gathered}
\mathbf{R}=\operatorname{Udiag}\left(\lambda_{1}, \lambda_{2}, \lambda_{3}\right) \mathbf{U}^{\dagger}=I \mathbf{U d i a g}\left(\hat{\lambda}_{1}, \hat{\lambda}_{2}, \hat{\lambda}_{3}\right) \mathbf{U}^{\dagger}, \\
{\left[\lambda_{1} \geqslant \lambda_{2} \geqslant \lambda_{3}, \quad \hat{\lambda}_{i} \equiv \lambda_{i} /(\operatorname{tr} \mathbf{R}), \quad(i=1,2,3)\right],}
\end{gathered}
$$

where $I=\operatorname{tr} \mathbf{R}$ is the intensity, $\mathbf{U}$ is the corresponding unitary diagonalization matrix, and $\lambda_{i}$ are the eigenvalues of $\mathbf{R}$, taken in decreasing order. Note that matrix $\mathbf{R}$ has the structure of a statistical covariance matrix of the zero-mean field variables and therefore the degree of polarimetric purity can be defined as $[8,12]$

$$
P_{3 \mathrm{D}}=\sqrt{\frac{1}{2}\left(3 \operatorname{tr} \hat{\mathbf{R}}^{2}-1\right)}, \quad(\hat{\mathbf{R}} \equiv \mathbf{R} / I),
$$

where $\operatorname{tr} \hat{\mathbf{R}}^{2}=\|\hat{\mathbf{R}}\|_{F}^{2},\|\hat{\mathbf{R}}\|_{F}$ being the Frobenius norm of $\hat{\mathbf{R}}$. This expression in terms of a metric of $\mathbf{R}$ highlights the interpretation of $P_{3 \mathrm{D}}$ as a distance of $\mathbf{R}$ from a fully random 3D state (represented by $\mathbf{R}_{u-3 \mathrm{D}} \equiv \mathbf{I} / 3$, I being the $3 \times 3$ identity matrix) [9].

An equivalent expression for $P_{3 \mathrm{D}}$ was introduced for the first time by Samson [13], and was also obtained later by Barakat [14] and by Gil [2] as the quadratic average of the relative differences between the eigenvalues of $\mathbf{R}$ :

$$
P_{3 \mathrm{D}}=\sqrt{\frac{1}{3} \sum_{\substack{i, j=1 \\ i<j}}^{3}\left(\hat{\lambda}_{j}-\hat{\lambda}_{i}\right)^{2} .}
$$

The ability of $P_{3 \mathrm{D}}$ to represent the degree of polarization (or degree of polarimetric purity) for electromagnetic waves, as well as some important features, has been studied by Setälä et al. [8,15-17] as well as by Gil et al. [2,12,18,19]. It is worth noting that the mathematical definition of $P_{3 \mathrm{D}}$ is, in fact, the $3 \mathrm{D}$ analog of the degree of polarimetric purity, $P_{4 \mathrm{D}}$, for the $4 \times 4$ Mueller matrices (depolarization index [20]) representing the transformation of polarization states by the action of a material medium [2]. The quantities $P_{3 \mathrm{D}}$ and $P_{4 \mathrm{D}}$ are $3 \mathrm{D}$ and $4 \mathrm{D}$ expressions of the following general $n$-dimensional formulation of the degree of purity for an $n \mathrm{D}$ coherency or density matrix $\boldsymbol{\Omega}[12,13]$ :

$$
P_{n \mathrm{D}}=\sqrt{\frac{1}{n-1}\left(\frac{n \operatorname{tr} \Omega^{2}}{\operatorname{tr}^{2} \Omega}-1\right)} .
$$

It should be stressed that, in the case of $2 \mathrm{D}$ representation, $P_{2 \mathrm{D}}$ is precisely the conventional 2D degree of polarization [19].

At this point, it is worth recalling that a generic concept of the $n \mathrm{D}$ polarization matrix can be applied in many different situations, like the superposition of $n$ pencils of electromagnetic radiation (second-order statistics of $n$ optical beams), which was formulated, very early on, by Wiener [21]. This notion was also considered (more specifically) by Barakat [22]. It is also well known that all the information contained in a Mueller matrix can be represented through the corresponding 4D coherency matrix [23]. Furthermore, the coherency matrix for a general electromagnetic field (with electric and magnetic vectors) is a $6 \mathrm{D}$ matrix (4D for beamlike fields). Other $n \mathrm{D}$ coherency matrices have been used, for instance, for the joint treatment of polarization and parity [24] and for the combined treatment of polarization and spatial coherence $[25,26]$. Thus the concept of an $n \mathrm{D}$ degree of purity expressed in Eq. (4) applies properly to $n \mathrm{D}$ coherency matrices or density matrices, and therefore it is of interest for many purposes in both optics and quantum physics.

It is also worth considering the following definitions, which will be particularly relevant for the aim of this work. Given a polarization matrix $\mathbf{R}$ it always can be represented by its corresponding intrinsic polarization matrix $\mathbf{R}_{O}$, defined as [27-29]

$$
\mathbf{R}_{O} \equiv \mathbf{Q}_{O} \mathbf{R} \mathbf{Q}_{O}^{T} \equiv\left(\begin{array}{ccc}
a_{1} & -i n_{O 3} & i n_{O 2} \\
i n_{O 3} & a_{2} & -i n_{1} \\
-i n_{O 2} & i n_{O 1} & a_{3}
\end{array}\right)
$$

where $\mathbf{Q}_{O}$ is the orthogonal matrix that diagonalizes the (symmetric) real part of $\mathbf{R}$ and $T$ denotes transpose. Thus $\mathbf{R}_{O}$ represents the same state of polarization as $\mathbf{R}$, but referred with respect to the corresponding intrinsic reference frame (hereafter denoted as $X_{O} Y_{O} Z_{O}$ ).

The intrinsic representation $\mathbf{R}_{O}$ makes it easier to identify and define the following concepts: (1) the degree of linear polarization $P_{l} \equiv \hat{a}_{1}-\hat{a}_{2}$ (with $\hat{a}_{i} \equiv a_{i} / I, i=1,2,3$ ), a measure of the sum of all contributions to linear polarization coming from the incoherent components $\mathbf{R}_{p}$ and $\mathbf{R}_{m}$ of $\mathbf{R}$; (2) the degree of circular polarization $P_{c} \equiv 2 \hat{n}_{O}$ (with $\hat{n}_{O} \equiv$ $\left.\sqrt{n_{O 1}^{2}+n_{O 2}^{2}+n_{O 3}^{2}} / I\right)$, a measure of the sum of all contributions to circular polarization coming from the incoherent components $\mathbf{R}_{p}$ and $\mathbf{R}_{m}$ of $\mathbf{R}$; and (3) the degree of directionality $P_{d}=1-3 \hat{a}_{3}$, a measure of how stable the plane is that contains the polarization ellipse [28]. These three quantities represent complementary contributions to $P_{3 \mathrm{D}}$, which can be written as [30]

$$
P_{3 \mathrm{D}}=\sqrt{\frac{3 P_{e}^{2}+P_{d}^{2}}{4}},
$$

where the degree of elliptical purity $P_{e} \equiv \sqrt{P_{l}^{2}+P_{c}^{2}}[10]$ summarizes all the contributions to polarimetric purity derived from the degrees of linear and circular purity (that is, all the contributions except for the purity due to the degree of directionality $P_{d}$ ).

To complete the necessary framework for our analysis, let us further recall that, among the different possible representations of $\mathbf{R}$ as compositions of mutually incoherent states, a privileged view of the structure of polarimetric purity of $\mathbf{R}$ is given by its 


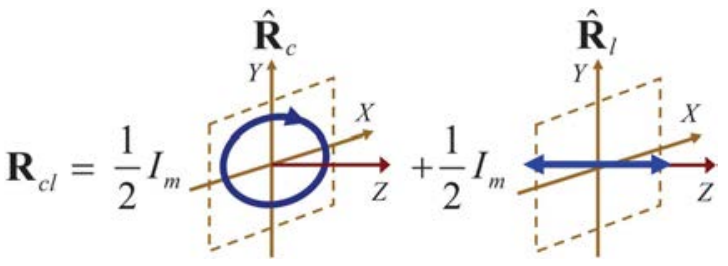

FIG. 1. A particular example of a nonregular state (with intensity $I_{m}$ ) is given by an equiprobable incoherent mixture $\mathbf{R}_{c l}$ of a circularly polarized state $\mathbf{R}_{c}$ and a linearly polarized state $\mathbf{R}_{l}$ whose electric field vibrates in the direction orthogonal to the plane containing the electric field of $\mathbf{R}_{c}$.

characteristic (or trivial) decomposition [2,10,18],

$$
\begin{gathered}
\mathbf{R}=I\left[P_{1} \hat{\mathbf{R}}_{p}+\left(P_{2}-P_{1}\right) \hat{\mathbf{R}}_{m}+\left(1-P_{2}\right) \hat{\mathbf{R}}_{u-3 \mathrm{D}}\right], \\
\hat{\mathbf{R}}_{p} \equiv \mathbf{U d i a g}(1,0,0) \mathbf{U}^{\dagger}, \hat{\mathbf{R}}_{m} \equiv \frac{1}{2} \mathbf{U d i a g}(1,1,0) \mathbf{U}^{\dagger}, \\
\hat{\mathbf{R}}_{u-3 \mathrm{D}} \equiv \frac{1}{3} \mathbf{I},
\end{gathered}
$$

where $\left(P_{1}, P_{2}\right)$ are the so-called indices of polarimetric purity of $\mathbf{R}[12,19]$ defined as

$$
P_{1}=\hat{\lambda}_{1}-\hat{\lambda}_{2}, \quad P_{2}=\hat{\lambda}_{1}+\hat{\lambda}_{2}-2 \hat{\lambda}_{3}=1-3 \hat{\lambda}_{3} .
$$

While the meanings of the pure and unpolarized components, $\hat{\mathbf{R}}_{p}$ and $\hat{\mathbf{R}}_{u-3 \mathrm{D}}$, respectively, are very clear as fully polarized and fully unpolarized states, the intermediate component $\hat{\mathbf{R}}_{m}$ involves, in a coupled manner, information on $P_{l}, P_{c}$, and $P_{d}$. In fact, polarization states can be classified into two types depending on whether $\hat{\mathbf{R}}_{m}$ is a real matrix (regular states) or not (nonregular states) [10].

In the case of regular states $\hat{\mathbf{R}}_{m}$ always represents a $2 \mathrm{D}$ unpolarized state. In other words, it can be considered an equiprobable mixture of two mutually orthogonal states whose polarization ellipses lie in the same plane, and therefore the polarization ellipse of $\hat{\mathbf{R}}_{m}$ evolves fully randomly but remains in a fixed plane. Thus, for regular states, $\hat{\mathbf{R}}_{m}$ does not contribute to either $P_{l}$ or $P_{c}$.

In the case of nonregular states, $\hat{\mathbf{R}}_{m}$ corresponds to an equiprobable mixture of two mutually orthogonal states whose polarization ellipses lie in different planes, and therefore $\hat{\mathbf{R}}_{m}$ does not represent a 2D unpolarized state, and exhibits nonzero contribution to $P_{c}$ and $P_{l}$ [10]. An example of a nonregular state (see Fig. 1) is any state $\mathbf{R}$ whose component $\hat{\mathbf{R}}_{m}$ is given by an equiprobable mixture $\hat{\mathbf{R}}_{c l}$ of a circularly polarized state and a linearly polarized state whose electric field vibrates in a direction that is orthogonal to the plane containing the polarization ellipse (circle) of the circular component [10].

In the case of states whose polarization ellipse remains in a constant plane during the measurement time (2D states), the characteristic decomposition of $\mathbf{R}$ takes the particular form [28]

$$
\mathbf{R}=I\left[P_{1} \hat{\mathbf{R}}_{p}+\left(1-P_{1}\right) \hat{\mathbf{R}}_{m}\right],
$$

where $\hat{\mathbf{R}}_{m}$ can always be considered a 2D unpolarized state, and the usual $2 \mathrm{D}$ degree of polarization is naturally defined as the ratio $I_{p} / I$ between the intensity $I_{p}$ of the pure component and the total intensity $I$. This fact has motivated some authors to extend this concept of degree of polarization to general (3D) polarization states [see Eq. (7)], and define it, in general, as the ratio $[2,6,31]$

$$
\begin{aligned}
\frac{I_{p}}{I} & =\frac{I_{p}}{I_{p}+I_{m}+I_{u-3 D}} \\
& =\frac{I P_{1}}{I P_{1}+I\left(P_{2}-P_{1}\right)+I\left(1-P_{2}\right)}=P_{1} .
\end{aligned}
$$

Obviously, the central idea that underlies this definition is to consider the degree of polarization as the relative portion of intensity of the pure component $\hat{\mathbf{R}}_{p}$, assuming that it contains all contributions to purity due to linear and circular polarization, and leaving aside the contributions associated with $P_{d}$. Such notion of degree of polarization assumes implicitly that $P_{1}=$ $P_{e}$, which holds exclusively for regular states. Furthermore, $P_{1}$ takes its minimum $P_{1}=0$ for states whose characteristic decomposition $\mathbf{R}=I\left[P_{2} \hat{\mathbf{R}}_{m}+\left(1-P_{2}\right) \hat{\mathbf{R}}_{u-3 \mathrm{D}}\right]$ lacks the pure component $\hat{\mathbf{R}}_{p}$, while $\hat{\mathbf{R}}_{m}$ retains some contribution $P_{2}$ to polarimetric purity, so that the value $P_{1}=0$ is not reached exclusively by fully random states $\left(\mathbf{R}_{u-3 \mathrm{D}}\right)$. It should be noted that the properties of $P_{1}$ and $P_{3 \mathrm{D}}$ as candidates for the 3D degree of polarization have been studied in [7,32], while an interesting comparative study of the role of the source coherence length on the properties of $P_{1}$ and $P_{3 \mathrm{D}}$ has been performed in [33].

From the above analysis we conclude that $P_{1}$ does not satisfy all the required properties to be associated to a 3D degree of polarimetric purity, because (a) in general $P_{1} \neq P_{e}$ (nonregular states) and therefore the contributions of $\hat{\mathbf{R}}_{m}$ to linear and circular degrees of polarization are not included in $P_{1}$ [10], and (b) the equality $P_{1}=0$ does not imply that necessarily $\mathbf{R}=$ $\mathbf{R}_{u-3 \mathrm{D}}$. Moreover, we also conclude that, unlike $P_{1}, P_{e}$ takes into account all the contributions to polarimetric purity due to the linear and circular degrees of polarization. Nevertheless, as occurs with $P_{1}$, the equality $P_{e}=0$ does not imply that $\mathbf{R}=\mathbf{R}_{u-3 \mathrm{D}}$. However, Eq. (6) instead shows that $P_{3 \mathrm{D}}$ accounts for all contributions to polarimetric purity, whether due to directionality or to linear and circular degrees of polarization.

In summary, the quantity that accounts for all contributions to purity, excluding that due to directionality, is the degree of elliptical purity $P_{e}$, and not $P_{1}$. In fact, $P_{e}$ includes the contributions to linear and circular purity, not only due to $\mathbf{R}_{p}$, but also due to $\mathbf{R}_{m}$. In other words, only for the regular states does the pure component of $\mathbf{R}$ contain all contributions to polarimetric purity, as given by $P_{e}$, and therefore $P_{e}$ cannot, in general, be considered as the relative intensity of a pure component of $\mathbf{R}$. In addition, value $P_{e}=0$ is not unique to fully random 3D states (characterized by $P_{3 \mathrm{D}}=0$ ). Concerning $P_{3 \mathrm{D}}$, it contains all contributions (linear, circular, directional) to polarimetric purity and can thus be considered a proper degree of polarimetric purity. The fact that the inequality $P_{3 \mathrm{D}} \geqslant 1 / 2$ holds for $2 \mathrm{D}$ states $[2,8,32]$ is nothing else than a natural consequence of the fact that they are characterized by $P_{d}=1$. Obviously, when assumed that a given polarization state is a 2D state $\left(P_{d}=1\right)$, the conventional 2D degree of polarization $P_{1}=P_{e}=P_{2 \mathrm{D}}$ is a consistent and appropriate measure of the contributions to polarimetric purity, beyond the one due to the deterministic directionality. This reinforces the advocacy for $P_{n \mathrm{D}}$ (with $n=2,3$ ) as the proper general parameter describing 
the degree of polarimetric purity of an electromagnetic wave (at the point in the space considered). The polarization matrix of any 3D field can straightforwardly be measured by using nanoprobe scatterers $[34,35]$ enabling an experimental determination of the concepts presented in this work.
This work was supported by MINECO (Grant No. FIS201458303-P), the Academy of Finland (Projects No. 268480 and No. 268705), and the Joensuu University Foundation. A.N. especially acknowledges the Swedish Cultural Foundation in Finland for financial support.
[1] C. Brosseau, Fundamentals of Polarized Light: A Statistical Optics Approach (Wiley, New York, 1998).

[2] J. J. Gil, Polarimetric characterization of light and media, Eur. Phys. J. Appl. Phys. 40, 1 (2007).

[3] E. Wolf, Coherence properties of partially polarized electromagnetic radiation, Nuovo Cimento 13, 1165 (1959).

[4] L. Mandel and E. Wolf, Optical Coherence and Quantum Optics (Cambridge University Press, Cambridge, 1995).

[5] J. C. Samson, Comments on polarization and coherence, J. Geophys. 48, 195 (1980).

[6] J. Ellis, A. Dogariu, S. Ponomarenko, and E. Wolf, Degree of polarization of statistically stationary electromagnetic fields, Opt. Commun. 248, 333 (2005).

[7] C. Brosseau and A. Dogariu, Symmetry properties and polarization descriptors for an arbitrary electromagnetic wavefield, Progress in Optics, edited by E. Wolf (Elsevier, Amsterdam, 2006), Vol. 49, Chap. 4.

[8] T. Setälä, A. Shevchenko, M. Kaivola, and A. T. Friberg, Degree of polarization for optical near fields, Phys. Rev. E 66, 016615 (2002).

[9] A. Luis, Degree of polarization for three-dimensional fields as a distance between correlation matrices, Opt. Commun. 253, 10 (2005).

[10] J. J. Gil, A. T. Friberg, T. Setälä, and I. San José, Structure of polarimetric purity of three-dimensional polarization states, Phys Rev. A 95, 053856 (2017).

[11] J. J. Gil and R. Ossikovski, Polarized Light and the Mueller Matrix Approach (CRC Press, Boca Raton, FL, 2016).

[12] J. J. Gil, J. M. Correas, P. A. Melero, and C. Ferreira, Generalized polarization algebra, Monogr. Semin. Mat. Garcia Galdeano 31, 161 (2004). Available from http://www.unizar.es/galdeano/ actas_pau/PDFVIII/pp161-167.pdf.

[13] J. C. Samson, Descriptions of the polarization states of vector processes: applications to ULF magnetic fields, Geophys. J. R. Astron. Soc. 34, 403 (1973).

[14] R. Barakat, Degree of polarization and the principal idempotents of the coherency matrix, Opt. Commun. 23, 147 (1977).

[15] T. Setälä, M. Kaivola, and A. T. Friberg, Degree of Polarization in Near Fields of Thermal Sources: Effects of Surface Waves, Phys. Rev. Lett. 88, 123902 (2002).

[16] T. Setälä, A. Shevchenko, M. Kaivola, and A. T. Friberg, Near-field degree of polarization, Proc. SPIE 4829, 1075 (2003).

[17] T. Setälä, K. Lindfors, and A. T. Friberg, Degree of polarization in 3D optical fields generated from a partially polarized plane wave, Opt. Lett. 34, 3394 (2009).
[18] J. J. Gil and I. San José, 3D polarimetric purity, Opt. Commun. 283, 4430 (2010).

[19] I. San José and J. J. Gil, Invariant indices of polarimetric purity. Generalized indices of purity for $n \times n$ covariance matrices, Opt. Commun. 284, 38 (2011).

[20] J. J. Gil and E. Bernabéu, Depolarization and polarization indices of an optical system, Opt. Acta 33, 185 (1986).

[21] N. Wiener, Generalized harmonic analysis, Acta Math. 55, 117 (1930).

[22] R. Barakat, $n$-fold polarization measures and associated thermodynamic entropy of $N$ partially coherent pencils of radiation, Opt. Acta 30, 1171 (1983).

[23] J. J. Gil, Characteristic properties of Mueller matrices, J. Opt. Soc. Am. A 17, 328 (2000).

[24] K. H. Kagalwala, G. Di Giuseppe, A. F. Abouraddy, and B. E. A. Saleh, Bell's measure in classical optical coherence, Nat. Photonics 7, 72 (2013).

[25] A. F. Abouraddy, What is the maximum attainable visibility by a partially coherent electromagnetic field in Young's double-slit interference?, Opt. Express 25, 18320 (2017).

[26] C. Okoro, H. E. Kondakci, A. F. Abouraddy, and K. C. Toussaint, Demonstration of an optical-coherence converter, Optica 4, 1052 (2017).

[27] M. R. Dennis, Geometric interpretation of the three-dimensional coherence matrix for nonparaxial polarization, J. Opt. A: Pure Appl. Opt. 6, S26 (2004).

[28] J. J. Gil, Interpretation of the coherency matrix for threedimensional polarization states, Phys. Rev. A 90, 043858 (2014).

[29] J. J. Gil, Intrinsic Stokes parameters for 3D and 2D polarization states, J. Eur. Opt. Soc.: Rapid Publ. 10, 15054 (2015).

[30] J. J. Gil, Components of purity of a three-dimensional polarization state, J. Opt. Soc. Am. A 33, 40 (2016).

[31] J. Ellis and A. Dogariu, On the degree of polarization of random electromagnetic fields, Opt. Commun. 253, 257 (2005).

[32] J. C. Petruccelli, N. J. Moore, and M. A. Alonso, Two methods for modeling the propagation of the coherence and polarization properties of nonparaxial fields, Opt. Commun. 283, 4457 (2010).

[33] J. M. Auñón and M. Nieto-Vesperinas, On two definitions of the three-dimensional degree of polarization in the near field of statistically homogeneous partially coherent sources, Opt. Lett. 38, 58 (2013).

[34] J. Ellis and A. Dogariu, Optical Polarimetry of Random Fields, Phys. Rev. Lett. 95, 203905 (2005).

[35] L.-P. Leppänen, A. T. Friberg, and T. Setälä, Partial polarization of optical beams and near fields probed with a nanoscatterer, J. Opt. Soc. Am. A 31, 1627 (2014). 